\title{
Discours
}

Revue de linguistique, psycholinguistique et

informatique. A journal of linguistics, psycholinguistics and computational linguistics

9| 2011

Varia

\section{Description sémantique de dans un premier temps : de la composition syntagmatique au discours}

\section{Catherine Schnedecker et Myriam Bras}

\section{OpenEdition}

Journals

Édition électronique

URL : http://journals.openedition.org/discours/8563

DOI : $10.4000 /$ discours. 8563

ISSN : 1963-1723

Éditeur :

Laboratoire LATTICE, Presses universitaires de Caen

\section{Référence électronique}

Catherine Schnedecker et Myriam Bras, «Description sémantique de dans un premier temps : de la composition syntagmatique au discours », Discours [En ligne], 9 | 2011, mis en ligne le 20 décembre 2011, consulté le 02 mai 2019. URL : http://journals.openedition.org/discours/8563 ; DOI : 10.4000/ discours.8563

\section{(c) (i) (9)}

Discours est mis à disposition selon les termes de la licence Creative Commons Attribution - Pas d'Utilisation Commerciale - Pas de Modification 4.0 International. 

Revue de linguistique, psycholinguistique et informatique

\section{Description sémantique de dans un premier temps : de la composition syntagmatique au discours}

Catherine Schnedecker

Université de Strasbourg, EA1339 LiLPa-Fonctionnements discursifs et traduction

Myriam Bras

Université de Toulouse, UMR5263 CLLE-ERSS

Catherine Schnedecker et Myriam Bras, «Description sémantique de dans un premier temps: de la composition syntagmatique au discours», Discours [En ligne], 9 | 2011, mis en ligne le 20 décembre 2011.

URL: http://discours.revues.org/8563. Consulté le 20 décembre 2011.

Titre du numéro: Varia

Coordination: Anne Le Draoulec et Josette Rebeyrolle

revues.org CENTRE POUR L'ÉDITION ÉLECTRONIQUE OUVE
CENTRE For OPEN ELECTRONIC PUBLISHING

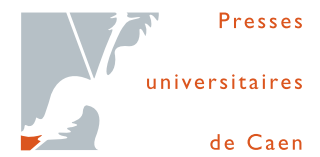

de Caen 



\title{
Description sémantique de dans un premier temps : de la composition syntagmatique au discours
}

\author{
Catherine Schnedecker \\ Université de Strasbourg, EA1339 LiLPa-Fonctionnements discursifs et traduction \\ Myriam Bras \\ Université de Toulouse, UMR5263 CLLE-ERSS
}

Cet article est consacré à l'expression adverbiale réputée sérielle dans un premier temps. Y est d'abord décrit le comportement sémantique de la locution dont le sens reste, pour une bonne partie, compositionnel, étant donné l'importance de la dimension temporelle et les contraintes qu'elle fait peser sur la nature des constituants de la configuration. Ensuite, nous montrons que l'expression dans un premier temps n'est «sérielle» que dans une partie de ses emplois. Dans les autres, non signalés dans la littérature, dans un premier temps marque une relation d'altérité qui peut être «faible» (i. e. encore teintée de temporalité) ou «forte» (i. e. nettement contradictoire).

Mots clés: marqueur sériel, marqueur d'intégration linéaire, relations temporelles, relation partie/tout, structures énumératives, discours, élaboration, contraste

The aim of this paper is to describe the semantic behavior of the French expression dans un premier temps, known as a serial marker. We show how its meaning is partly compositional, given the importance of the temporal dimension and the constraints which this places on the nature of the constituents. We then show that dans un premier temps is "serial" in only some of its uses. In others, not reported in the literature, dans un premier temps expresses a relation of "otherness" which can be either "weak" (i.e. keeping a temporal value), or "strong" (i.e. sharply contradictory).

Keywords: serial marker, temporal relations, part-whole relations, enumerative structures, discourse relations, elaboration, contrast

\section{Introduction ${ }^{1}$}

De prime abord, les adverbiaux dits ordinaux (premièrement, en premier lieu et dans un premier temps) n'ont rien de bien mystérieux. Régulièrement construits sur la base d'adjectifs ordinaux, ils forment un paradigme net sur le double plan sémantique et fonctionnel. Comme leurs adjectifs de base, ils indiqueraient, vu leur rapport avec la numération, «le rang dans une série» (Guimier, 1996: 126) et ordonneraient, par voie de conséquence, la matière discursive, servant au «balisage textuel» (Riegel, Pellat et Rioul, 1994: 380). De là, leur appartenance à la classe des

1. Les auteurs remercient les relecteurs anonymes et les responsables du numéro pour leurs remarques et leurs critiques qui ont permis d'améliorer la qualité de l'article. 
marqueurs d'intégration linéaire (Turco et Coltier, 1988: 57) dont les membres, disent les auteurs, «accompagnent l'énumération sans fournir de précision autre que le fait que le segment discursif qu'ils introduisent est à intégrer de façon linéaire dans la série». C'est effectivement ce qu'ils semblent faire en [I] :

[I] Je crois que j'avais fait en même temps trois découvertes: premièrement les adultes avaient un «travail», deuxièmement ce travail des adultes était une chose capitale, auprès de laquelle rien de ce que j'avais appris auparavant ne comptait, et troisièmement, j'ignorais quelle était cette chose capitale en ce qui concernait notre père. (Guimier, 1996: 126)

En outre, les segments ainsi corrélés sont dans un étroit rapport d'interdépendance, ce que souligne Nøjgaard (1995) en les qualifiant de «relationnels sériels». Ce lien d'interdépendance tiendrait, comme il est dit souvent, au caractère «fondamentalement anaphorique» de cette classe d'adverbes (voir Guimier, 1996: I25-I26; Molinier, $\left.1990^{2}\right)$. De fait, l'occurrence de premièrement en [I], tout comme celle de dans un premier temps en [2a], laisse attendre un fragment subséquent ${ }^{3}$ :

[2a] Le député de Toul étant mort, une élection partielle est organisée. Dans un premier temps, la seule candidature républicaine est celle d'un modéré, qui se prononce contre l'amnistie.

(V. Hugo, Depuis l'exil, ז885)

Pour autant, ces éléments de description ne sont pas réellement satisfaisants pour quatre raisons, au moins. D'une part, ils ne rendent pas justice au paradigme des adverbiaux ordinaux qui comprend, outre les adverbes proprement dits ${ }^{4}$, les locutions dans un premier temps et en premier lieu qui varient au double plan nominal (lieu vs temps) et prépositionnel (en vs dans), se distinguant ainsi de leurs correspondants ordinaux en -ment. D'autre part, ils n'aident à distinguer ni leur valeur sémantique propre ni leur rôle, pas plus qu'ils n'aident à les dissocier d'autres familles de marques réputées sérielles, elles aussi, comme d'abord/ensuite/enfin. Or, force est de constater que ces marques ne se substituent pas impunément les unes aux autres, en témoignent les manipulations de [2a] dont résultent des degrés d'irrecevabilité variables, $[2 \mathrm{~b}]$ étant meilleur que $[2 \mathrm{c}]$ et $[2 \mathrm{~d}]$ :

[2b] ?Le député de Toul étant mort, une élection partielle est organisée. D’abord, la seule candidature républicaine est celle d'un modéré, qui se prononce contre l'amnistie.

2. Pour qui la classe des conjonctifs, dont participent les ordinaux, «rassemble des adverbes définis par l'inaptitude à figurer dans l'énoncé initial d'un discours, ou, de façon équivalente, par l'exigence d'un contexte gauche spécifique» (Molinier, 1990: 30).

3. D'autant qu'en [I] l'amorce trois découvertes est claire sur les intentions du locuteur.

4. Auxquels il conviendrait d'ajouter leur variante - s'il s'agit bien d'une variante - primo, secondo, etc. et les suites de chiffres. 
[2c] * Le député de Toul étant mort, une élection partielle est organisée. Premièrement, la seule candidature républicaine est celle d'un modéré, qui se prononce contre l'amnistie.

[2d] * Le député de Toul étant mort, une élection partielle est organisée. En premier lieu, la seule candidature républicaine est celle d'un modéré, qui se prononce contre l'amnistie.

Par ailleurs, ils occultent le fait que ces unités peuvent renvoyer à l'ordre, extralinguistique, des événements, ce que suggère d'ailleurs très explicitement le nom temps dans dans un premier temps en [2a], autant qu'à celui que confère à son propre discours le locuteur du discours. Enfin, les questions de la fréquence, de la primauté, etc. des différents emplois de ces marques sont passées sous silence alors qu'une simple interrogation de FRANTEXT révèle des disparités étonnantes (voir tableau I).

\begin{tabular}{|l|l|l|l|}
\hline $\begin{array}{l}\text { Nombre d'occurrences selon } \\
\text { l'ordinal }\end{array}$ & Lieu & Temps & Adverbe en -ment \\
\hline premier X & $\mathrm{I} 254$ & $\mathrm{I} 04$ & $\mathrm{I} 559$ \\
\hline Date de la I ${ }^{\text {re }}$ occurrence & $\mathrm{I} 537$ & $\mathrm{I} 876$ & $\mathrm{I} 534$ \\
\hline second X & 887 & $2 \mathrm{I}$ & $64 \mathrm{I}$ \\
\hline Date de la I ${ }^{\text {re }}$ occurrence & $\mathrm{I} 549$ & $\mathrm{I} 9 \mathrm{II}$ & $\mathrm{I} 508$ \\
\hline deuxième X & 25 & 27 & $\mathrm{I} 87$ \\
\hline Date de la $\mathrm{I}^{\text {re }}$ occurrence & $\mathrm{I} 902$ & $\mathrm{I} 957$ & $\mathrm{I} 766$ \\
\hline troisième X & $\mathrm{I} 30$ & 2 & $\mathrm{I} 23$ \\
\hline Date de la I ${ }^{\text {re }}$ occurrence & $\mathrm{I} 560$ & $\mathrm{I} 844$ & $\mathrm{I} 655$ \\
\hline quatrième X & $\mathrm{I} 7$ & $\mathrm{O}$ & $5 \mathrm{I}$ \\
\hline Date de la I ${ }^{\text {re }}$ occurrence & $\mathrm{I} 560$ & & $\mathrm{I} 655$ \\
\hline dernier X & 364 & 2 & $\mathrm{I0} 33^{5}$ \\
\hline Date de la I ${ }^{\text {re }}$ occurrence & $\mathrm{I} 624$ & $\mathrm{I} 965$ & $\mathrm{I} 558$ \\
\hline Total occurrences & 2677 & $\mathrm{I} 56$ & 3594 \\
\hline
\end{tabular}

Tableau 1: nombre d'occurrences dans FRANTEXT de en (premier/second/deuxième/troisième/ quatrième/dernier) lieu; dans un (premier/second/deuxième/troisième/quatrième/dernier) temps; (premièrement/secondement/deuxièmement/troisièmement/quatrièmement/dernièrement)

5. Dernièrement est à part, puisqu'il s'emploie comme adverbe temporel non sériel, dès les premiers emplois. Il mériterait une étude en soi. 
En effet, les emplois de dans un premier temps et des autres marqueurs de la série «dans un $x^{e}$ temps» sont à la fois nettement moins nombreux que ceux de en premier lieu (en second/... / $x^{e}$ lieu) ou ceux de premièrement (deuxièmement/... / xemement), et ce, dans un rapport qui va de I à I7 ou de I à 23. Ils sont, en outre, beaucoup plus récents que ceux de ses congénères, puisqu'ils remontent au $\mathrm{XIX}^{\mathrm{e}}$ siècle alors que les deux autres sont attestés dès le XVI ${ }^{e}$ siècle.

Partant de là, notre objectif consistera à étudier dans un premier temps (désormais $D P T$ ) pour en dégager les différents emplois, que nous analyserons sous l'angle, d'une part, du sémantisme des composants lexicaux (l'adjectif ordinal et le nom temps) qui sera détaillé en temps utile, et, d'autre part, d'un statut d'adverbial changeant ${ }^{6}$. En filigrane, nous poserons quelques jalons susceptibles de faire le départ entre $D P T$ et ses prétendus jumeaux en premier lieu et premièrement déjà cités, mais aussi d'abord et à première vue ${ }^{7}$.

Compte tenu du faible nombre d'occurrences de DPT collectées dans FRANTEXT (I04), nous avons élargi notre collection d'exemples à des extraits de Wikipédia (310), de L'Est républicain (482) et du Monde (508), portant à I 400 le nombre d'extraits analysés.

\section{DPT: une locution figée...}

Sur le plan catégoriel, le paradigme des adverbiaux construits sur la base de DPT présente la plupart des caractéristiques des locutions figées. Nous examinons ci-dessous les transformations possibles sur chacun des éléments de la locution.

L'adjectif ordinal est obligatoire et ne supporte pas la suppression (voir exemple [3b]) - alors que certaines expressions admettent la construction prép. + dét. + temps «nu» (exemples [4] et [5]) -, pas plus qu'il ne tolère le remplacement par un autre adjectif $(\text { exemple }[3 \mathrm{c}])^{8}$.

6. Cette étude est la première d'une série où nous envisageons : i) les configurations de discours accueillant $D P T$ et la modélisation des relations de discours impliquées dans le cadre de la Théorie des représentations discursives segmentées (SDRT) (cf. Bras et Schnedecker, soumis); ii) la comparaison entre DPT et en premier lieu (cf. Bras et Schnedecker, à paraître); iii) l'évolution comparée du couple d'adverbiaux (cf. Bras et Schnedecker, en préparation). L'analyse de DPT pourra ensuite être facilement étendue à l'ensemble du paradigme des formes construites sur dans $+u n+$ ordinal + temps.

7. Voir Lenepveu (à paraître) que nous remercions très chaleureusement pour sa relecture attentive.

8. En outre, DPT ne change pas de nombre et l'expression dans les premiers temps, qui semblerait constituer une exception - d'autant qu'elle se paraphrase également par au début -, ne présente pas tout à fait les mêmes conditions d'emplois syntaxiques et sémantiques:

Il s'installa finalement à San Francisco et ne revint plus en France, ne donnant quasiment pas de nouvelles pendant dix ans. Dans les premiers temps, ma mère enquêta fébrilement pour savoir si son fils était ou non homosexuel, ce qui la dérangeait davantage que mon père.

(G. Bouillier, Rapport sur moi, 2002) 
[3a] La physiologie des glandes mammaires évolue en deux temps. Dans un premier temps, les glandes se développent pour être prêtes à assurer la production de lait. Dans un deuxième temps, facultatif celui-là, se produit la sécrétion lactée, au décours des accouchements [...].

(Encyclopédie médicale Quillet, 1965)

[3b] * La physiologie des glandes mammaires évolue en deux temps. Dans un temps, les glandes se développent pour être prêtes à assurer la production de lait.

[4] Avec le temps, il a fini par avoir son permis de conduire.

[5] Dans le temps, il roulait à bicyclette.

[3c] * La physiologie des glandes mammaires évolue en deux temps. Dans un premier temps, les glandes se développent pour être prêtes à assurer la production de lait. Dans un autre temps, facultatif celui-là, se produit la sécrétion lactée, au décours des accouchements [...].

Le nom se laisse substituer, mais dans des conditions particulières qui sont la nonappartenance à une série (exemple [6]), ou la présence d'une amorce du type dét. $+\mathrm{N}_{\mathrm{t}}$ (voir [3d]). En l'absence d'amorce du type dét. $+\mathrm{N}_{\mathrm{t}}$, la commutation est plus difficile ([7a] et [7b]). Dans le cas de non-appartenance à une série, les équivalents nominaux tels que phase ou moment se rencontrent le plus souvent avec des compléments du nom (exemple [8]). Le nom ne se laisse pas supprimer en seconde mention (voir [3e]) ${ }^{9}$.

[6] La certitude est acquise grâce à l'épochè. Dans un premier moment, la sympathie, la conscience de soi trouvait son fondement dans son être pour-autrui [...].

(J. Vuillemin, L'être et le travail, 1949)

[3d] La physiologie des glandes mammaires évolue en deux phases. Dans une première phase, les glandes se développent pour être prêtes à assurer la production de lait. Dans une deuxième phase, facultative celle-là, se produit la sécrétion lactée [...].

[7a] [...] je lui souris et je tentai de retirer mon bras mais elle le serra avec détermination, sans me regarder, menant résolument la marche. Dans un premier temps, j'ai songé qu'elle voulait que je l'accompagne chez elle, mais peu à peu la situation me parut plus étrange, puis inquiétante.

(C. Legendre et J. Bonnetto, Photobiographies, 2007)

9. Dans l'exemple:

[...] s'effectuent en deux temps : au premier est liée l'hydratation des cations échangeables, et c'est autour de ces ions hydratés que viendraient dans un deuxième temps se fixer les molécules organiques. (S. Caillère et S. Hénin, Minéralogie des argiles, 1963)

qu'on pourrait qualifier d'exception à première vue, le SN le premier ne participe pas à une locution adverbiale de type prép. + dét. + temps, mais est un argument du prédicat être lié à. 
[7b] ?? [...] je lui souris et je tentai de retirer mon bras mais elle le serra avec détermination, sans me regarder, menant résolument la marche. Dans une première phase, j’ai songé qu'elle voulait que je l'accompagne chez elle, mais peu à peu la situation me parut plus étrange, puis inquiétante.

[8] Sa barbe longue, ses cheveux emmêlés, son regard fiévreux le masquent, mais un cœur fidèle ne devrait-il pas pressentir et savoir? Dans un premier moment de désespoir, il s'est laissé dépasser, mais bien vite il court les rejoindre.

(M. Barrès, Un jardin sur l'Oronte, 1922)

[3e] * La physiologie des glandes mammaires évolue en deux temps. Dans un premier, les glandes se développent pour être prêtes à assurer la production de lait. Dans un deuxième, facultatif celui-là, se produit la sécrétion lactée, au décours des accouchements $[\ldots]$.

La préposition semble sujette à changements et se laisse remplacer par en (exemple [9]) ou à (exemple [ı]), voire supprimer (exemple [ı。]), ce qui entraîne, parfois, la suppression du déterminant (exemple [II]), mais ces changements modifient le sens de l'adverbial.

[9] [...] pour que les intéressés eux-mêmes se sentent peu à peu confirmés dans un droit qu'au début ils n'osaient même pas concevoir. En un premier temps, limitées, puis élargies, les réalisations laissent encore hors de leur champ de larges fractions de la population, les moins éprouvées ou les moins armées.

(F. Perroux, L'économie du XX'e siècle, 1964)

[Io] L'homme devait venir à bout des douze rats, uniquement avec ses dents; un premier temps, les rats s'effrayaient de la grosseur carnassière de l'intrus; au deuxième temps, ils attaquaient, ils déchiquetaient joues, oreilles, nez, poitrine.

(J.-L. Bory, Un prix d'excellence, 1979)

[II] Il faut maintenant décomposer ces mouvements. Ils se font en deux temps. Premier temps, les épaules se haussent, la poitrine se dilate et s'emplit d'air; deuxième temps, les épaules s'abaissent, la poitrine se vide.

(Alain, Propos, 1936)

Nous avons relevé dans notre corpus quelques cas de commutations et d'adjonctions. Le déterminant indéfini peut par exemple commuter avec le défini (en [I2]), le nom admettre des modifieurs adnominaux (voir [I3] et [I4]) ou adjectivaux (exemple [I5]), et l'adjectif lui-même se laisser spécifier par tout (exemple [I6]).

[i2] Dans un premier temps les chloroplastes isolés et entiers, fonctionnent sans illumination, en l'absence de [...]. Dans le second temps, les granums, partie verte, étant enlevés, l'opération est poursuivie à l'obscurité, en présence de [...].

(Histoire générale des sciences, 1964) 
[i3] Voilà donc, en fin de compte, ce que, dans un premier temps d'observation et de réflexion, nous révèle le spectacle du monde en mouvement. Mais est-ce vraiment bien tout $[\ldots]$.

(P. Teilhard de Chardin, Le phénomène bumain, 1955)

[I4] [...] pourquoi le footballeur est traité de manchot par les adeptes des autres sports. I. Prendre le ballon. Se rendre maître du ballon, c'est, dans un premier temps de contact, le contrôler pour l'utiliser aux meilleures fins de progression. Dans la maitrise de la balle, le joueur exprime ses qualités [...].

(J. Mercier, Le football, 1966)

[i5] Bref, elle l'investissait d'une manière on ne peut plus nette, flagrante, absolue, de cette fonction de «représentation de soi» que j’ai, dans un premier temps sommaire, accordée au «nounours».

(J. Roubaud, La boucle, 1993)

[16] Et à Jules absent de Paris, je mentis de même, dans un tout premier temps, par ce réflexe de l'omission.

(H. Guibert, À l'ami qui ne m'a pas sauvé la vie, 1990)

Mais ces exemples semblent constituer des exceptions ou des cas particuliers où la variante de $D P T$ ne fait pas partie d'une série, ce qui nous amène à la conclusion que l'emploi de $D P T$ n'est pas totalement libre.

\section{2. ...dont la valeur des constituants persiste}

Si $D P T$ constitue donc bien une expression figée, le sens de ses constituants persiste, d'une certaine manière, avec des incidences très nettes sur ses emplois, ce que nous montrerons en commençant par le $\mathrm{N}$ temps puis nous passerons à l'examen du rôle de l'adjectif ordinal.

\subsection{Temps dans DPT}

Le $\mathrm{N}$ temps connaît des emplois indéniablement massifs, comme dans [i7] par exemple, mais il est envisagé, dans $D P T$, sous un angle comptable, par un phénomène de conversion, déjà bien identifié (voir [18]), en raison notamment du déterminant indéfini :

[17] Nous avons passé (du/beaucoup de/un peu de) temps sur cet article.

[I8] J'ai mis du pinot dans mon baeckeofe. J'ai mis un pinot blanc/gris dans mon baeckeofe.

Cela étant, à la différence des phénomènes de conversion, les occurrences de temps ainsi constituées ne sont pas des sous-catégories de temps. Il s'agit plutôt d'entités temporelles qui présentent au moins quatre caractéristiques. 
Premièrement, elles sont bornées, ce que prouve leur équivalence avec les $\mathrm{N}$ phase (exemple [19]), période, intervalle, acte (exemple [20]) des SN-amorces, des reprises anaphoriques (voir [2I]) ou des syntagmes juxtaposés (voir [22]):

[19] Dès 1958 , on parvenait à fractionner le chloroplaste et à séparer matériellement les deux phases [...]. Dans un premier temps les chloroplastes isolés et entiers, fonctionnent sans illumination, en l'absence de [...]. Dans le second temps, les granums, partie verte, étant enlevés, l'opération est poursuivie à l'obscurité, en présence de [...].

(Histoire générale des sciences, 1964)

[2o] Le drame, en général, se déroulait en plusieurs actes. Dans un premier temps, tout le monde se laissait subjuguer par la beauté, le charme, la douceur des quatre sœurs. Et puis, peu à peu, ceux qui s'imaginaient comprendre et savoir - ce sont les mêmes, bien souvent, qui se trompent avec le plus de force - découvraient sous l'apparence une réalité qui leur faisait peur.

(J. d'Ormesson, Tous les bommes sont fous, 1986)

[2I] [...] il faut tout d'abord dissocier l'échantillon de façon à permettre la séparation des particules constitutives et, dans un deuxième temps, procéder à la séparation de ces dernières et à leur groupement en classes définies par des diamètres extrêmes. La première phase constitue [...].

(S. Caillère et S. Hénin, Minéralogie des argiles, 1963)

[22] - Oui... Il y a peut-être en effet contradiction. C'est vrai que, dans un premier temps, au cours d'une période pendant laquelle il n'avait pas l'intention de reconnaître la Chine, de Gaulle a souvent employé l'argument que vous [...].

(P. Mendès-France, CEuvres complètes, 1990)

Ensuite, elles s'inscrivent elles-mêmes dans un intervalle temporel, ce que prouvent les trois faits suivants:

- le fait que le SN-hôte de temps «nu» paraît incomplet (voir [23]) - les exemples [24], [25] et [26], proférés avec une intonation particulière, étant des exceptions:

[23] *(Dans) un temps $/ *$ (dans) un premier temps $\rightarrow$ un (premier) temps de quoi?

[24] Il y a un temps pour tout.

[25] Il me prêta son appartement pour un temps.

[26] Nous avons passé UN temps sur cet article! $\approx$ Nous avons passé un (long/*bref) temps sur cet article. 
- le fait qu'il appelle des modifieurs adjectivaux dimensionnels (exemple [27]) ${ }^{10}$ ou adnominaux (exemple [28]) ${ }^{11}$ :

[27] Nous avons passé un (certain) temps/un temps (relativement long/infini) sur cet article.

[28] L'interrogatoire du malade et de son entourage, dont la médecine hippocratique avait montré toute l'importance, constituait le premier temps de l'enquête.

(M. Bariéty et C. Coury, Histoire de la médecine, 1963)

- le fait que ces adnominaux appartiennent à la catégorie des accomplissements (exemple [28]), c'est-à-dire qu'ils renvoient à des entités temporelles ayant une durée (ce qui exclut les achèvements - voir [29]), bornées (ce qui exclut les activités - voir [30]), et hétérogènes, puisqu'elles doivent pouvoir se décomposer en sous-parties (ce qui exclut notamment les états $-\operatorname{voir}[3 \mathrm{I}])$ :

[29] * Le premier temps de l'explosion de la bombe.

[30] * ${ }^{*}$ Le premier temps du dessin.

[3I] * ${ }^{*}$ Le premier temps du savoir.

Troisièmement, leur extension temporelle n'est pas stable (Berthonneau, 1990), puisqu'elles ne dénotent pas de quantité temporelle fixe, à la différence de termes comme minute, heure, etc.; cette quantité n'est pas non plus spécifiable comme le suggère le contraste entre [32] et [33]:

[32] une durée de 2 heures $(\approx$ la durée $=2$ heures $)$

[33] *un temps de 2 heures $(\neq \text { le temps }=2 \text { heures })^{12}$

Enfin, l'intervalle ainsi dénoté n'est pas fixé sur l'axe temporel, ce que montre la différence entre les SN de [34] et [35]:

[34] un temps de l'enquête $\approx$ un moment de l'enquête

[35] le début/la fin de l'enquête $\approx$ le moment initial/final

\footnotetext{
10. Ou axiologiques: Nous avons passé un temps (précieux/incroyable/pas possible) sur cet article.

11. Les cas où un $\mathrm{SN}$ en temps renvoie cotextuellement à une proposition sont différents, comme on va le voir.

12. Mais 2 beures de temps.
} 


\subsection{Le rôle des ordinaux}

13. Qui parlent, l'un de «hiérarchie», l'autre de «rang», c'est-à-dire d'un ensemble d'unités lexicales fini, doté d'extrémités, lexicalisées, dans le cas des ordinaux, par le couple premier/dernier et dont les entités sont soumises à une relation orientée (Cruse, 1986), i. e. asymétrique (si x est premier/second/troisième, ... par rapport à y, y ne peut être premier/second/troisième, ... par rapport à x), transitive (si x précède $y$, si $\mathrm{y}$ précède $\mathrm{z}$, alors $\mathrm{x}$ précède $\mathrm{z}$ ) et connexe («étant donné deux termes quelconques de la classe à ordonner, l'un doit précéder et l'autre suivre» [Cruse, I986: II3-II4 et voir également 192-I95; cf. aussi Lyons, 1978: 233-236]).

14. Voir, chez Lakoff et Johnson (1985: chap. 9), les métaphores qui conceptualisent le temps comme un objet en mouvement. 


\subsection{Contraintes sur l'emploi de DPT}

Dans les SN de structure dét. +ordinal +temps, la nature du nom temps et le rôle partitif des adjectifs ordinaux premier, second, ... permettent l'établissement de relations de partie à tout relevant du domaine temporel. Elles s'établissent entre des parties décrites par les SN en temps et des touts de deux types:

- des collections d'entités de même nature, comme l'illustre [39] avec le SN-tout deux temps;

[39] = [3a] La physiologie des glandes mammaires évolue en deux temps. Dans un premier temps, les glandes se développent pour être prêtes à assurer la production de lait. Dans un deuxième temps, facultatif celui-là, se produit la sécrétion lactée, au décours des accouchements [...].

- un tout événementiel dont l'extension temporelle est découpée par les référents des SN décrivant les parties:

[40] La bile se recueille facilement chez l'homme grâce au tubage duodénal. Dans un premier temps on obtient un mélange de sécrétions biliaires et duodénopancréatiques, c'est la bile A. Dans un deuxième temps, après avoir introduit [...]. (Encyclopédie médicale Quillet, 1965)

Le tout événementiel peut être implicite et se laisser reconstruire au moment de l'interprétation. En [4I] par exemple, on reconstruit un tout correspondant à la conséquence de l'arrivée dans le foie des colonies amibiennes:

[4I] Mais à partir de ce foyer colique il arrive que des colonies amibiennes parviennent par voie sanguine au niveau du foie. Dans un premier temps il se produit autour des intrus une réaction inflammatoire hépatique puis sous l'afflux de globules blancs se forme une poche de pus.

(Encyclopédie médicale Quillet, 1965)

Les relations partie/tout en jeu sont donc de deux types: la première est une relation «élément/collection», la seconde une relation "portion/tout», selon la catégorisation des relations de partie à tout de Aurnague et Vieu (I993) que nous étendons ici aux entités temporelles ${ }^{15}$.

15. Dans la relation "élément/collection», les éléments sont groupés en collections sur la base de propriétés partagées (la forêt, la foule, les brebis du troupeau). Dans la relation "portion/tout», le tout peut-être une substance désignée par un terme massif (un verre d'eau, un litre de lait, un morceau de sucre) ou un nom comptable singulier ayant un équivalent massique (une tranche de gâteau/une tranche de ce gâteau, un morceau de fromage/un morceau de ce fromage). Les portions sont faites de la même substance que le tout et ne remplissent aucune fonction particulière par rapport au tout. Voir aussi Aurnague (2004: 50-63) ainsi que Vieu et Aurnague (2007). 
De l'idée que les SN de structure un + ordinal + temps signalent que les segments temporels ainsi dénotés entretiennent une relation de succession et d'altérité et qu'ils participent d'un tout, découlent certaines contraintes ${ }^{16}$ sur l'environnement discursif de DPT comme:

- l'impossibilité des emplois ex abrupto de DPT et son caractère d'adverbial «conjonctif» (voir supra);

- le caractère temporel particulier de sa structure de rattachement;

- son caractère dit «sériel».

\section{Typologie des emplois de DPT}

Les observations précédentes expliquent, en effet, que $D P T$ participe des «relationnels sériels», ainsi qu'il a été dit en introduction, sans pour autant se réduire à cet usage. De fait, ses configurations d'accueil se scindent en quatre sous-classes, dépendant, en partie, de ce caractère sériel ou non.

\section{1. $\quad D P T$ et les relations partie / tout événementielles}

Une première série d'emplois de $D P T$, illustrée par [39] et [40] ci-dessus et par [42] et [43] ci-dessous, se caractérise, du point de vue formel, par le fonctionnement sériel homogène du marqueur, qui est généralement suivi de dans un (second/deuxième) temps, les séries à trois termes ou plus étant, pour ainsi dire, inexistantes, dans notre corpus tout du moins:

[42] Dans cette conjoncture nouvelle, les Américains prennent très au sérieux leur rôle de patron du capitalisme international. Dans un premier temps, ils conseillent aux pays européens de décoloniser à l'amiable. Dans un deuxième temps, ils n'hésitent pas à proclamer d'abord le respect puis le soutien du principe: l'Afrique aux Africains. (F. Fanon, Les damnés de la terre, 196I)

[43] La technique utilisée consiste, dans un premier temps, à drainer la fistule et, dans un deuxième temps, à la mettre à plat sans blesser le sphincter anal, car le grand risque dans cette affection, c'est l'incontinence sphinctérienne.

(Encyclopédie médicale Quillet, 1965)

Dans ces exemples, les SN un premier temps/un deuxième temps font référence à une partie de l'extension temporelle du tout événementiel auquel réfère l'énoncé précédent $D P T$, ainsi que nous l'avons indiqué en section 2.3. De telles relations partie/tout apparaissent aussi dans des segments de discours articulés par des séries hétérogènes comme l'illustre [44]:

16. Les deux dernières contraintes étant également associables aux autres adverbes de la série: dans un second temps, dans un deuxième temps, dans un troisième temps... 
[44] $=[20]$ Le drame, en général, se déroulait en plusieurs actes. Dans un premier temps, tout le monde se laissait subjuguer par la beauté, le charme, la douceur des quatre sœurs. Et puis, peu à peu, ceux qui s'imaginaient comprendre et savoir - ce sont les mêmes, bien souvent, qui se trompent avec le plus de force - découvraient sous l'apparence une réalité qui leur faisait peur.

(J. d'Ormesson, Tous les hommes sont fous, 1986)

Dans cet emploi, $D P T$ se rattache à la catégorie syntaxique des adverbes de phrase, malgré le fait qu'il puisse être extrait par c'est... que (comme en [45]). En effet, il figure en tête de propositions principales (voir [39], [40] et [42]) ou subordonnées (exemple [46]) et se laisse détacher en tête de phrase négative (exemple [47]):

[45] C'est dans un premier temps que les follicules ne produiront plus d'ovule.

[46] Les possibilités de polymérisation des nucléotides déjà existants [...] ont été examinées et on s'est rendu compte que dans un premier temps on pouvait aisément les convertir en nucléosides phosphates cycliques, lesquels sont polymérisés quantitativement dans un deuxième temps par action du tétraphényl pyrophosphate à température ordinaire.

(M. Privat de Garilhe, Les acides nucléiques, 1963)

[47] Que se passe-t-il donc au cours de la ménopause? Entre 45 et 55 ans, le plus souvent autour de la cinquantaine, les ovaires atteints de vieillissement sont peu à peu envahis par la sclérose et perdent leur valeur fonctionnelle. Dans un premier temps, [les follicules ne produiront plus d'ovule. Ils sécréteront en abondance de la folliculine et à l'absence de l'ovulation correspond une absence de la sécrétion de l'autre hormone femelle de la deuxième partie du cycle menstruel, la lutéine. La conséquence en sera la perturbation des cycles menstruels, tantôt très courts, tantôt très longs, avec alternance de règles hémorragiques ou minimes.] Dans un deuxième temps les ovaires ne peuvent même plus sécréter de folliculine: la carence est alors totale. La glande hypophyse réagit: devant l'absence d'hormones sexuelles femelles, les hormones stimulantes sexuelles hypophysaires gonadotrophines sont sécrétées en abondance, mais en vain, puisque les ovaires ne peuvent plus répondre. L'excès de gonadotrophine est responsable de bon nombre des troubles de la ménopause.

(Encyclopédie médicale Quillet, 1965)

Sa portée peut s'étendre sur plusieurs segments, ainsi que l'illustre l'exemple [47], où il joue le rôle d'introducteur de cadre de discours, au sens de Charolles (1997), propriété qu'il partage avec nombre d'adverbiaux détachés en position initiale, comme les marqueurs d'intégration linéaire (Schnedecker, 2001a; Jackiewicz, 2002) ou les adverbiaux de localisation temporelle (Le Draoulec et Péry-Woodley, 2005).

Il semble participer des adverbiaux temporels du fait qu'il renvoie explicitement à la notion de temps et qu'il se laisse remplacer par des SP ou des adverbiaux dénotant la temporalité avec une idée de durée (voir [48b]): 
[48a] = [3a] La physiologie des glandes mammaires évolue en deux temps. Dans un premier temps, les glandes se développent pour être prêtes à assurer la production de lait. Dans un deuxième temps, facultatif celui-là, se produit la sécrétion lactée, au décours des accouchements.

[48b] La physiologie des glandes mammaires évolue en deux temps. (Au début/d'abord/au départ/pendant un (certain) [temps/un moment]) les glandes se développent pour être prêtes à assurer la production de lait.

Mais, paradoxalement, il se soustrait au test clé permettant l'identification des adverbiaux temporels: la possibilité de répondre à une question en quand (Molinier et Lévrier, 2000: 240; Nøjgaard, 1995: 327) ${ }^{17}$, voire pendant combien de temps même s'il supporte le clivage (voir supra), signifiant qu'il est susceptible d'assumer un rôle rhématique dans la phrase ${ }^{18}$ :

[49a] Et si Bonne-Maman, dans un premier temps, «joue aux absences», «fait l'égarée», excelle dans le rôle de «l'abandonnée», très vite ses absences ne sont plus jouées [...]. (Y. Navarre, Biographie, 198I)

[49b] Quand Bonne Maman a-t-elle joué aux absences? $-{ }^{*}$ Dans un premier temps.

Du point de vue discursif, DPT s'inscrit dans des configurations discursives relativement élaborées, initiées fréquemment par un SP-amorce du type en $x$ temps, comme l'illustre [48a] ci-dessus. Ce SP-amorce ne marque d'ailleurs pas tant le temps que la manière. En effet, il suit généralement le verbe, peut servir de réponse à une question en comment (exemple [5o]) et, enfin, se laisse paraphraser par d'une manière + adj., comme par exemple dans [5I]:

[5०] Comment évolue-t-elle? Elle évolue en deux temps.

[5I] Elle évolue en deux temps $\approx$ d'une manière progressive/progressivement.

Dans ce type de configuration, $D P T$ a pour remplaçants les adverbiaux de la série d'abord, ensuite, puis (voir [48c]):

[48c] La physiologie des glandes mammaires évolue en deux temps. D’abord, les glandes se développent pour être prêtes à assurer la production de lait. Puis, [il] se produit la sécrétion lactée, au décours des accouchements.

17. Au même titre, selon l'auteur, qu’un jour, une fois, désormais, auparavant, ensuite, depuis, dorénavant, naguère.

18. Voir l'explication que donne Nøjgaard (1995: 327-328) à l'échec de l'épreuve de quand: seul un tout petit nombre se déroberait au test de quand, par incapacité à assumer un rôle rhématique dans la phrase. 
$D P T$ figure, le plus souvent, à l'initiale des segments introduits, tout comme ses congénères, et introduit le plus souvent des propositions. Sa présence est facultative, à condition toutefois que dans un deuxième temps soit remplacé par un connecteur qui permet de séparer les phases, tel puis (en [48e]), a fortiori lorsque le contenu des propositions est contradictoire ${ }^{19}$, ce qui s'exprime, le cas échéant, par le sémantisme antonymique des prédicats (se hausser/s'abaisser en [52]):

[48d] ?La physiologie des glandes mammaires évolue en deux temps. Les glandes se développent pour être prêtes à assurer la production de lait. Dans un deuxième temps, facultatif celui-là, se produit la sécrétion lactée, au décours des accouchements.

[48e] La physiologie des glandes mammaires évolue en deux temps. Les glandes se développent pour être prêtes à assurer la production de lait. Puis se produit la sécrétion lactée, au décours des accouchements.

[52a] Voilà donc le rire, pris en gros. Il faut maintenant décomposer ces mouvements. Ils se font en deux temps. Premier temps, les épaules se baussent, la poitrine se dilate et s'emplit d'air; deuxième temps, les épaules s'abaissent, la poitrine se vide.

(Alain, Propos, 1936)

[52b] ?[Ces mouvements] se font en deux temps. Les épaules se baussent, la poitrine se dilate et s'emplit d'air; les épaules s'abaissent, la poitrine se vide.

[52c] [Ces mouvements] se font en deux temps. Les épaules se baussent, la poitrine se dilate et s'emplit d'air; puis les épaules s'abaissent, la poitrine se vide.

Sur le plan sémantique, ces segments contiennent la plupart du temps des prédicats téliques au sens de Garey (1957), et décrivent des événements, entités bornées temporellement (Kamp, 1979). Dans le cas de procès atéliques comme les activités ou les états (Vendler, 1967), DPT requiert un aspect verbal perfectif, qui borne l'éventualité, en créant un événement sur le plan discursif (Kamp et Reyle, 1993). C'est le cas en [53a], exemple forgé pour les besoins de la démonstration, où le passé composé borne le procès, qui contraste avec [53b] où l'imparfait laisse le procès non borné, qui devient un état sur le plan discursif, ou avec [54a] qui décrit également des états sur le plan discursif:

[53a] J'ai passé une mauvaise période l'hiver dernier. Dans un premier temps, j’ai été malade, une sorte de bronchite dont j’ai eu du mal à me guérir. Dans un second temps, j’ai eu des pannes de voiture à répétition, j'arrivais tout le temps en retard au boulot.

19. Voir Bras, Le Draoulec et Vieu (200I) pour une explication du rôle de puis rendant possible l'établissement d'une relation de contraste dans de tels cas. 
[53b] *J'ai passé une mauvaise période l'hiver dernier. Dans un premier temps, j'étais malade, une sorte de bronchite dont j’ai eu du mal à me guérir. Dans un second temps, j'avais des pannes de voiture à répétition, j'arrivais tout le temps en retard au boulot.

[54a] *Je n'irai pas au cinéma ce soir. Dans un premier temps, j’ai du boulot; dans un second temps, je suis fatiguée.

Cela distingue, par parenthèses, DPT de premièrement et de d'abord dont les constituants n'ont pas besoin de participer d'un tout événementiel et peuvent introduire des états (exemples $[54 \mathrm{~b}]$ et $[54 \mathrm{c}])^{20}$ :

[54b] Je n'irai pas au cinéma ce soir. Premièrement, j’ai du boulot; deuxièmement je suis fatiguée.

[54c] Je n'irai pas au cinéma ce soir. D’abord, j’ai du boulot; ensuite je suis fatiguée.

Enfin, les segments introduits par $D P T$ ne permutent pas sans altérer la valeur de vérité de l'énoncé (exemples [55a] et [55b]):

[55a] = [43] La technique utilisée consiste, dans un premier temps, à drainer la fistule et, dans un deuxième temps, à la mettre à plat sans blesser le sphincter anal [...].

[55b] \#La technique utilisée consiste, dans un premier temps, à mettre à plat la fistule sans blesser le sphincter anal et, dans un deuxième temps, à la drainer.

C'est cet emploi de $D P T$ qui participe de la structuration métadiscursive telle qu'évoquée en introduction. En effet, dans la mesure où les actes de discours constituent des «processus hétérogènes», décomposables en phases, il n'est pas étonnant que $D P T$ puisse structurer le développement discursif, comme l'illustre [56] :

[56] Nous nous proposons d'exposer ici cette théorie officielle. Dans un premier temps, nous en rappellerons les bases concrètes et l'argumentation scientifique. Dans un deuxième temps nous reprendrons les faits et tâcherons de les réinterpréter. (adapté de F. Fanon, Les damnés de la terre, 196I)

Cela étant, ce type d'emploi est assez rare, dans notre corpus du moins, ce qui là encore distingue $D P T$ des adverbiaux ordinaux dont c'est, au contraire, la fonction primordiale (voir Schnedecker, 2oora et b).

Nous retiendrons de cette section que, dans les séquences discursives présentant les caractéristiques qui viennent d'être évoquées, $D P T$ est le premier d'une série de marqueurs introduisant des segments qui développent ou spécifient le contenu sémantique de la proposition ( $\mathrm{p}-\mathrm{I}$ ) qui précède la série. Il contribue à l'établissement

20. Voir Bras (2007) pour une analyse de d'abord et Schnedecker (2oora et b) pour une étude de premièrement. 
d'une relation partie/tout événementielle, le tout étant décrit par la proposition qui précède $(\mathrm{p}-\mathrm{I})$, et la partie par la ou les propositions qui sont dans sa portée.

\section{2. $D P T$ et la temporalité}

Un deuxième type d'emploi de $D P T$ se manifeste formellement d'une double manière. Soit il apparaît au sein de séries d'adverbiaux hétérogènes en ce qu'elles instancient d'autres marqueurs adverbiaux à ordinaux comme en second lieu, ou des expressions d'autres séries à caractère "temporel» telles que puis (voir [57] et [58]) ou ensuite $(\operatorname{voir}[59])^{21}$, soit la série est «incomplète» comme en [60] où dans un second temps n'est pas précédé de $D P T$.

[57] [...] mais comme si le gel avait figé dans une sorte de métallique immobilité les rouages de l'énorme machine qui, dans un premier temps, s'était mise en branle, puis s'était tout à coup arrêtée, comme quelque mécanisme soudain grippé, ou comme si les mécanos s'étaient aperçus d'un oubli [...].

(C. Simon, Les Géorgiques, 198I)

$[58]=[4 \mathrm{I}]$ Mais à partir de ce foyer colique il arrive que des colonies amibiennes parviennent par voie sanguine au niveau du foie. Dans un premier temps il se produit autour des intrus une réaction inflammatoire hépatique puis sous l'afflux de globules blancs se forme une poche de pus.

(Encyclopédie médicale Quillet, 1965)

[59] c) microsomes: fractions microsomales de réticulocytes. Selon les expériences de Rabinovitz et Olson, une suspension de réticulocytes est marquée dans un premier temps par des amino-acides. On isole ensuite la fraction microsomale de ces cellules et on incube avec du jus cellulaire frais.

(M. Privat de Garilhe, Les acides nucléiques, 1963)

[6o] Les bras tendus des cavaliers formaient des lances qui visaient l'adversaire au visage et qui dans un second temps se transformaient en harpons, crochaient le cavalier au col et le tiraient sur le côté ou en arrière.

(M. Tournier, Le roi des Aulnes, 1970)

Ce type de configuration présente deux caractéristiques.

21. Cela étant, force est de constater que les marqueurs d'autres séries s'ajoutent à la série dans un premier/second temps plus qu'ils ne la structurent, comme le montrent les énoncés suivants, ce qui tendrait à prouver que $D P T$ ne marquerait pas (que?) la succession temporelle :

[...] les malades ne doivent pas s'étonner si dans un premier temps on leur fait faire une radio de l'estomac et du côlon pour éliminer toute atteinte de ces organes, puis dans un deuxième temps une radiographie des voies biliaires.

(Encyclopédie médicale Quillet, 1965)

Ils conduisent à des diplômes de niveau divers: Cap, Bp, Bei ou Bec; enfin, dans un second temps, aux diplômes de technicien et technicien supérieur puis au diplôme d'ingénieur.

(Encyclopédie pratique de l'éducation, 1960) 
Formellement, les marqueurs appartiennent à des segments dont les catégories grammaticales sont identiques, qu'il s'agisse de propositions complètes ([58] et [59]) ou elliptiques (exemple [6I]), de verbes à l'infinitif (exemple [62]), de SN (exemple [63]) ou de SP (exemple [64]), et qui respectent souvent le principe du parallélisme syntaxique:

[6I] La loi imposant dorénavant de libeller la correspondance administrative en arabe, il est probable que celle-ci sera rédigée en français dans un premier temps puis traduite en arabe.

(Le Monde, 1998)

[62] Un déficit important est donc prévisible à moyen terme, s'inquiètent les experts, qui préconisent de fixer dans un premier temps à deux cents le quota de places offertes au concours d'internat en anesthésie-réanimation, puis de porter le nombre à trois cent cinquante en cinq ans.

(Le Monde, 1998)

[63] La direction de Bosch a annoncé au comité d'entreprise, le lundi 4 mai, sa décision d'implanter à Rodez sa nouvelle chaîne de fabrication de l'injecteur pompes Diesel (PDE), un investissement de 500 millions de francs qui générera 250 emplois dans un premier temps et éventuellement 400 par la suite si, comme prévu, le nombre d'injecteurs produits chaque jour passe progressivement de 3000 à 6000 .

(Le Monde, 1998)

[64] Conduite, dans un premier temps, par le président du FLNKS, Roch Wamytan, puis par son prédécesseur à la tête de l'organisation indépendantiste, Paul Néaoutyine, elle devait encore rencontrer François Bayrou, président du groupe UDF de l'Assemblée, jeudi, peu avant l'ouverture de la séance.

(Le Monde, 1998)

Sémantiquement, ces segments satisfont l'exigence d'altérité imposée par DPT grâce à un simple changement dans les arguments du prédicat, qui expriment alors d'autres acteurs, d'autres chiffres, d'autres modalités d'expression.

Cet emploi de $D P T$ se distingue du précédent par l'absence, dans la proposition antérieure à l'adverbial, de SN dénotant un tout événementiel. Ce tout se laisse récupérer ou reconstruire à la rigueur, comme nous l'avons vu pour [58], mais ses composants n'ont pas le même statut que dans le cas d'une relation partie/tout événementielle, au sens où ils ne constituent pas les étapes obligatoires au (bon) déroulement d'un processus. C'est ce qu'illustre [63] dont le second segment comprend l'adverbial «éventuellement».

C'est pourquoi nous dirons ici que $D P T$ marque une succession temporelle, dont la seule contrainte tient, comme dit plus haut, à l'expression d'une forme d'altérité. 


\section{3. $D P T$ : de la temporalité à l'altérité}

Le troisième emploi de $D P T$ n'est plus sériel, comme dans les deux cas précédents, puisque le marqueur se manifeste «hors-série», étant le seul adverbial «de son espèce».

On distingue alors deux cas de figure. Dans le premier cas, le segment introduit par DPT fonctionne en tandem avec un autre, qui peut être introduit par la locution avant de/que (exemples [65] à [67]) ${ }^{22}$ ou qui régit une proposition comparative (exemples [68] à [70]):

[65] Les quatre nouveaux indices seront calculés à partir du 26 février et diffusés, dans un premier temps, en écus et en dollars, avant de l'être en euros et en dollars.

(Le Monde, 1998)

[66] Dans un premier temps, le lancement permettra, dès la mi-septembre, de tester en Ile-de-France le succès des magasins, avant d'étendre la formule sur la France entière.

(Le Monde, 1998)

[67] Direction et syndicats sont dans un premier temps solidaires face à l'extérieur, avant que chacun ne retrouve son rôle.

(Le Monde, 1998)

[68] Les deux foyers distincts et le fonctionnement presque simultané des détecteurs de fumée correspondent plus au scénario d'un incendie volontaire quà celui de l'accident et du court-circuit électrique évoqués dans un premier temps.

(Le Monde, 1998)

[69] L'UDF a dû préciser, vendredi, qu'elle avait suspendu six personnes de plus qu'elle ne l'avait annoncé dans un premier temps, jeudi.

(Le Monde, 1998)

[70] Il apparaît également que le déplacement d'Augusto Pinochet était loin d'être aussi privé que l'a soutenu Londres dans un premier temps.

(Le Monde, 1998)

Dans le second cas de figure, DPT apparaît dans des constituants dépendants syntaxiquement:

- des propositions relatives dont le temps grammatical (le plus-que-parfait) marque l'antériorité par rapport à l'événement dénoté dans la principale (exemples [7r] à [73]):

22. Le Draoulec (2008) montre que dans de tels cas avant que/de a un rôle de connecteur temporel et peut être remplacé par puis. Même s'ils ne sont pas formellement sériels, ces cas peuvent être rapprochés de ceux qui ont été étudiés en section 3.2 où $D P T$ fait couple avec puis. 
[71] D'ailleurs, un premier revers a déjà contraint la Food and Drug Administration à suspendre, l'an dernier, des essais cliniques [qu'elle avait, dans un premier temps, approuvés,] rappelle Jonathan Allan.

(Le Monde, 1998)

[72] Aussitôt, le conseil d'administration de More Group, [qui avait, dans un premier temps, recommandé à l'unanimité l'offre de Clear Channel à ses actionnaires,] revient sur sa position.

(Le Monde, 1998)

[73] La tentative d'intimidation n'a, semble-t-il, pas eu de répercussions sur la mission, mais des salariés de la caisse, [qui avaient dans un premier temps accepté de répondre aux questions des enquêteurs,] n'ont pas désiré confirmer leurs dires.

(Le Monde, 1998)

- des constructions détachées (exemples [74] à [76]):

[74] Limitée dans un premier temps aux pays dits émergents la Thaillande, la Malaisie, les Philippines et l'Indonésie, la crise a atteint Hong Kong et Taïwan, puis a déstabilisé la Corée du Sud et le Japon, deux grandes puissances de l'économie mondiale. (Le Monde, 1998)

[75] Amer dans un premier temps, il ne tarde pas à relancer sa croisade. (Le Monde, 1998)

[76] Estimé dans un premier temps entre $\mathbf{1 9 0}$ et 220 francs, le prix de l'action a été arrêté à 205 francs, après consultation des investisseurs potentiels.

(Le Monde, 1998)

Dans les deux cas, le contenu du segment introduit par DPT entretient une relation d'altérité et/ou de contradiction avec son segment-recteur, $D P T$ ayant justement pour effet de signaler que le changement/revirement est décalé dans le temps, sans rien préciser pour autant des date, durée et intervalle, ce qui permet d'éviter les incohérences.

Trois propriétés permettent de distinguer ces emplois des précédents. D’abord, $D P T$ n'occupe pas - ou rarement - la position frontale, mais reste juxtaposé à l'unité qu'il modifie. En l'occurrence, il peut suivre ou précéder l'adjectif (voir [75] et [77a]), ou s'intercaler entre l'auxiliaire et le participe passé des verbes (voir [72] et [73]). Bref, il n'est plus tant adverbe de phrase qu'adverbe de constituant.

Ensuite, il n'admet pas toujours les mêmes remplaçants que précédemment. Il se montre plus rétif à d'abord ${ }^{23}$ (voir [77b] et [78b]) mais admet, en revanche, plus aisément au départ, initialement, au préalable ou à l’origine (voir [78c]):

23. Sauf si d'abord est à l'initiale: d'abord amer, ... 
[77a] Direction et syndicats sont dans un premier temps solidaires face à l'extérieur, avant que chacun ne retrouve son rôle.

(Le Monde, 1998)

[77b] ?Direction et syndicats sont d'abord solidaires face à l'extérieur, avant que chacun ne retrouve son rôle.

[78a] Amer dans un premier temps, il ne tarde pas à relancer sa croisade. (Le Monde, 1998)

[78b] ?Amer d'abord, il ne tarde pas à relancer sa croisade.

[78c] Amer au départ, il ne tarde pas à relancer sa croisade.

Enfin, $D P T$ n'introduit plus expressément des processus hétérogènes, mais peut modifier des états (voir être solidaire en [77], être amer en [78]).

\section{4. $\quad D P T$ et la correction argumentative}

Dans le dernier type de configuration repéré, $D P T$ n'est pas non plus sériel, mais fait couple, d'une certaine façon, avec un connecteur contre-argumentatif (mais, cependant). De ce fait, les contenus propositionnels ainsi mis en relation entretiennent une relation sémantique contradictoire que manifeste très clairement l'impossibilité de supprimer les marqueurs ${ }^{24}$ :

[79a] Dans un premier temps, les deux fédérations avaient pourtant eu dans l'idée d'installer leurs traditionnelles agapes dans la salle polyvalente du Stade de France, mais elles ont reculé en apprenant les tarifs de location (900000 francs pour 2000 mètres carrés).

(Le Monde, 1998)

[8oa] Dans un premier temps, le groupe arabe à l'ONU avait présenté un projet de résolution condamnant Israël, mais les Européens les ont encouragés à accepter un compromis, qui permettait d'adopter un texte commun avec les États-Unis. (Le Monde, 1998)

[8ia] Il souhaitait dans un premier temps revendre American Sky Broadcast à PrimeStar, mais le département de la justice américain s'était opposé à ce projet.

(Le Monde, 1998)

[79b] * Les deux fédérations avaient pourtant eu dans l'idée d'installer leurs traditionnelles agapes dans la salle polyvalente du Stade de France, elles ont reculé en apprenant les tarifs de location ( 900000 francs pour 2000 mètres carrés).

24. L'impossibilité étant entendue dans le sens où la suppression entraîne soit l'incohérence du discours, soit une tout autre interprétation. 
[8ob] * Le groupe arabe à l'ONU avait présenté un projet de résolution condamnant Israël, les Européens les ont encouragés à accepter un compromis, qui permettait d'adopter un texte commun avec les États-Unis.

[8Ib] *Il souhaitait revendre American Sky Broadcast à PrimeStar, le département de la justice américain s’était opposé à ce projet.

$D P T$ retrouve ici un rôle de constituant de phrase, avec davantage de mobilité que dans les cas vus plus haut, dans la mesure où il ne figure pas systématiquement à l'initiale des propositions qu'il introduit ${ }^{25}$. Cet emploi se reconnaît notamment à certains aspects du contenu propositionnel du segment sur lequel porte DPT:

- la présence d'adverbes de modalité (probablement en [82]),

[82] Fondements scientifiques du «New Deal»: Roosevelt hésita d'abord et, dans un premier temps, il crut probablement que l'Amérique pourrait être sortie de ses difficultés par une politique de type orthodoxe. C'était en tout cas le thème [...].

(P. Mendès-France, Euvres complètes, 1986)

- la présence de verbes d'attitude propositionnelle (vouloir, soubaiter), ou encore de prédicats verbaux comme conclure (exemple [83]), croire (exemple [84]), ou avoir l'idée, qui signalent le caractère virtuel de ces contenus propositionnels, ceux qui sont introduits par le connecteur adversatif ayant au contraire un statut factuel indéniable:

[83] Cette division des groupes nationaux en «situations politiques» conduit à conclure dans un premier temps que toutes les combinaisons sont imaginables. Cependant, $\grave{a}$ y regarder de plus près, on constate que si toutes les combinaisons s'appliquaient [...]. (H. Carrère d'Encausse, L'empire éclaté, 1978)

[84a] Dans un premier temps, le président du Conseil avait cru identifier ce document comme l'un des procès-verbaux figurant au dossier de la conférence. Mais lorsque [...]. (P. Mendès-France, Euvres complètes, 1986)

$D P T$ change alors de «remplaçant» et se substitue, le cas échéant, à de prime abord/à première vue ${ }^{26}$ :

25. DPT peut d'ailleurs, comme dans le premier type d'emploi étudié, avoir une portée étendue à plusieurs segments et jouer le rôle d'introducteur de cadre:

Et les Bourses occidentales ont fini par décrocher et, avec elles, les sicav d'actions. Dans un premier temps, [ces dernières avaient pourtant largement profité de la tempête sur les marchés financiers émergents. Les capitaux, en particulier, qui avaient fui l'Asie, étaient venus se réfugier en masse à Wall Street et sur les places boursières européennes, jugées indestructibles.] Mais l'intensification de la tourmente financière, avec le décrochage du rouble, et les craintes que celle-ci ne provoque un ralentissement économique brutal en Occident, ou ne dégénère en crise du système bancaire, ont grippé [...]. (Le Monde, 1998)

26. V. Lenepveu nous signale que le remplacement de à première vue par DPT est possible uniquement lorsque à première vue semble encore régi par des verbes de perception. Lorsqu'il marque un point de 
[84b] À première vue/De prime abord, le président du Conseil avait cru identifier ce document comme l'un des procès-verbaux figurant au dossier de la conférence. Mais lorsque $[\ldots]$.

\section{Conclusion}

Au terme de cette étude, nous aurons essayé de faire valoir deux aspects importants des adverbiaux du type de $D P T$.

Le premier a trait au rôle que continuent à jouer les constituants de $D P T$, en dépit du figement. À cet égard, le sens de $D P T$ reste, pour une bonne partie, compositionnel, étant donné l'importance de la dimension temporelle et des contraintes qu'elle fait peser sur la nature des constituants de la configuration. C'est d'ailleurs une différence flagrante qui sépare, sur le plan morpho-sémantique et discursif, DPT de son concurrent le plus proche morphologiquement, en premier lieu (voir Bras et Schnedecker, à paraître), et qui est la conséquence directe de l'histoire des formes. En tant que «dernier arrivé» dans le paradigme des ordinaux adverbiaux (voir supra), DPT laisse encore transparaitre le sémantisme de base de ses constituants.

Deuxièmement, il ressort de notre analyse que $D P T$ n'est «sériel» que dans une partie de ses emplois: ceux où il relève d'une relation temporelle de type «partie/tout» et ceux que nous avons qualifiés de «temporels» stricts.

D'autres emplois, non signalés dans la littérature, sont apparus, où $D P T$ marque une relation d'altérité qui peut être «faible», c'est-à-dire encore teintée de temporalité, ou «forte», c'est-à-dire nettement contradictoire. Les différences entre les différents emplois répertoriés sont synthétisées dans le tableau 2 (voir infra ${ }^{27}$.

Ce dernier aspect appelle d'ailleurs toute une série de questions qui ouvrent la voie à des études relatives à l'histoire du marqueur ${ }^{28}$. Comment se répartissent, sur la faible diachronie de $D P T$, ses emplois: suivent-ils le tracé dégagé par les études sur la grammaticalisation en partant des emplois temporels pour évoluer vers un sens plus abstrait, l'expression de la contradiction argumentative, ou coexistent-ils dès le départ?

C'est dire si ces formes, apparemment anodines et au sémantisme évident, se révèlent, au bout du compte, infiniment plus riches et énigmatiques qu'elles n'en ont l'air...

vue comme dans $\grave{A}$ première vue, cette jeune fille a une quinzaine d'années, DPT n'est plus possible.

27. Dans Bras et Schnedecker (soumis), nous analysons les configurations discursives accueillant DPT dans ses différents emplois, nous prolongeons la présente étude en mettant au jour les relations de discours auxquelles $D P T$ est associé : élaboration avec son contexte gauche, narration ou contraste avec son contexte droit.

28. Voir Bras et Schnedecker (à paraître). 


\begin{tabular}{|c|c|c|c|c|}
\hline & \multicolumn{2}{|c|}{ Temporalité } & \multicolumn{2}{|c|}{ Altérité } \\
\hline & $\begin{array}{l}\text { Relation } \\
\text { événementielle } \\
\text { partie/tout }\end{array}$ & $\begin{array}{l}\text { Absence de } \\
\text { relation } \\
\text { partie/tout }\end{array}$ & Faible & $\begin{array}{l}\text { Forte } \\
\text { (contradiction } \\
\text { argumentative) }\end{array}$ \\
\hline $\begin{array}{l}\text { Statut de } \\
\text { l'adverbial }\end{array}$ & $\begin{array}{l}\text { adverbe } \\
\text { de phrase }\end{array}$ & ambigu & $\begin{array}{l}\text { adverbe } \\
\text { de constituant }\end{array}$ & $\begin{array}{l}\text { adverbe } \\
\text { de phrase }\end{array}$ \\
\hline $\begin{array}{l}\text { Position } \\
\text { dans la } \\
\text { phrase }\end{array}$ & initiale & $\begin{array}{l}\text { postposé au } \\
\text { constituant } \\
\text { modifié }\end{array}$ & & initiale \\
\hline $\begin{array}{l}\text { Présence } \\
\text { obligatoire }\end{array}$ & - & - & + & + \\
\hline $\begin{array}{l}\text { Éléments } \\
\text { corrélés }\end{array}$ & $\begin{array}{l}\text { série homogène } \\
\text { ou hétérogène }\end{array}$ & $\begin{array}{l}\text { série hétérogène } \\
\text { ou trouée }\end{array}$ & $\begin{array}{l}\text { hors-série } \\
\text { avant que/ } \\
\text { connecteurs } \\
\text { comparatifs }\end{array}$ & $\begin{array}{l}\text { connecteurs } \\
\text { contre- } \\
\text { argumentatifs }\end{array}$ \\
\hline $\begin{array}{l}\text { Présence } \\
\text { d'un SN- } \\
\text { amorce }\end{array}$ & + & - & - & - \\
\hline Substituts & d'abord & d'abord & $\begin{array}{l}\text { au départ/ } \\
\text { initialement/ } \\
\text { au préalable / à } \\
\text { l'origine }\end{array}$ & $\begin{array}{l}\text { de prime abord/à } \\
\text { première vue }\end{array}$ \\
\hline
\end{tabular}

Tableau 2: récapitulatif des emplois de $D P T$

\section{Bibliographie}

Aurnague, M. 2004. Les structures de l'espace linguistique. Louvain - Paris: Peeters.

Aurnague, M. et Vieu, L. 1993. A Three-Level Approach to the Semantics of Space. In C. Zelinsky-Wibbelt (ed.), The Semantics of Prepositions: From Mental Processing to Natural Language Processing. Berlin - New York: Mouton de Gruyter: 395-439.

Berthonneau, A.M. 199o. Site déictique et site anaphorique. Étude stratigraphique des compléments de temps. In G. KLEIBER et J.-E. TyvaERT (eds), L'anaphore et ses domaines. Paris: Klincksieck: $\mathrm{I}-50$.

Bras, M. 2007. French Adverb d'abord and Discourse Structure. In M. Aurnague, J.-M. Larrazabal et K. Korta (eds), Language, Representation and Reasoning. Memorial Volume to Isabel Gómez Txurruka. Bilbao: Universidad del País Vasco: 77-Ioz.

Bras, M., Le Draoulec, A. et Vieu, L. 200I. French Adverbial Puis between Temporal Structure and Discourse Structure. In M. BRAs and L. VIEU (eds), Semantic and Pragmatic Issues in Discourse and Dialogue: Experimenting with Current Theories. CRiSPI series 9. Amsterdam - New York - Paris: Elsevier: I09-I46. 
Bras, M. et Schnedecker, C. soumis. French Adverbial dans un premier temps and Discourse Relations: From Elaboration to Contrast. Actes du colloque LPTS, Paris, 2I-23 septembre 2009 .

Bras, M. et SCHNEDECKer, C. à paraître dans Langue française. Dans un (premier + second $+n^{\text {ieme})}$ temps vs en (premier + second $+n^{\text {ieme}}$ ) lieu: qu'est ce qui fait la différence? Actes du «Colloque de linguistique française et de didactique du français: l'expression de l'espace et du temps en français: quelles formes pour quels sens?», Belgrade, 23-26 mars 20II.

Bras, M. et Schnedecker, C. en préparation. Dans un (premier + second $\left.+n^{\text {ieme }}\right)$ temps vs en

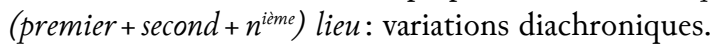

Charolles, M. 1997. L'encadrement du discours - univers, champs, domaines et espaces. Cabiers de recherche linguistique 6: $\mathrm{I}^{-} 73$.

Cruse, D.A. 1986. Lexical Semantics. Cambridge: Cambridge University Press.

Garey, H. 1957. Verbal Aspect in French. Language 33 (2): 9I-IIO.

Guimier, C. 1996. Les adverbes du français. Le cas des adverbes en -ment. Paris - Gap: Ophrys.

JACKIEWICZ, A. 2002. Repérage et délimitation des cadres organisationnels pour la segmentation automatique des textes. In Y. Toussaint et C. Nedellec (eds), CIFT'200z: colloque international sur la fouille de texte. Actes du colloque d'Hammamet, 2I-23 octobre 2002. Le Chesnay: INRIA : 95-107.

Kamp, H. 1979. Events, Instants and Temporal Reference. In R. BäueRle, U. EgLI et A. von Stechow (eds), Semantics from Different Points of View. Berlin - New York: Springer-Verlag.

Kamp, H. et Reyle, U. 1993. From Discourse to Logic. Dordrecht - Boston - Londres: Kluwer Academic Publishers.

Lakoff, G. et Jonhson, M. 1985. Les métaphores de la vie quotidienne. Paris : Éditions de Minuit.

Le Draoulec, A. 2008. Il retombe puis rebondit avant de retomber à nouveau...: puis et avant que dans la progression narrative. In M. BiRKELUND, M.-B.M. HANSEN et C. NorÉn (eds), L'énonciation dans tous ses états. Mélanges offerts à Henning Nølke. Berne: Peter Lang: 403-432.

Le Draoulec, A. et Péry-Woodley, M.-P. 2005. Encadrement temporel et relations de discours. Langue française I48: 45-60.

LENEPVEu, V. à paraitre. À première vue, un marqueur d'anticipation textuel. Journal of French Language Studies.

Lyons, J. 1978. Éléments de sémantique. Paris: Larousse.

Molinier, C. 1990. Une classification des adverbes en -ment. Langue française 88: 28-40.

Molinier, C. et LÉvrier, F. 2000. Grammaire des adverbes en -ment. Genève - Paris: Droz.

NøJGAARD, M. 1995. Les adverbes français. Essai de description fonctionnelle. Historisk-filosofiske Meddelelser 66-3. Copenhague: Munksgaard. T. 3.

Riegel, M., Pellat, J.-C. et Rioul, R. 1994. Grammaire méthodique du français. Paris: PUF.

SCHNEDECKER, C. 2oora. Adverbes ordinaux et introducteurs de cadre: aspects linguistiques et cognitifs. Linguisticae Investigationes 24 (2): 257-287. 
Schnedecker, C. 2oorb. De l'ordre dans les adverbes: les ordinaux. In C. BuRidant, G. Kleiber et J.-C. Pellat (eds), Par monts et par vaux, itinéraires linguistiques et grammaticaux: mélanges de linguistique générale et française offerts au professeur Martin Riegel. Louvain: Peeters: $365-378$.

Turco, G. et Coltier, D. I988. Des agents doubles de l'organisation textuelle: les marqueurs d'intégration linéaire. Pratiques 57: 57-79.

Vendler, Z. 1967. Verbs and Times. In Linguistics in Philosophy. Ithaca - New York: Cornell University Press: 97-I2I.

Vieu, L. et Aurnague, M. 2007. Part-of Relations, Functionality, Dependence. In M. Aurnague, M. Hickmann et L. Vieu (eds), The Categorization of Spatial Entities in Language and Cognition. Human cognitive processing 20. Amsterdam - Philadelphia: John Benjamins : 307-336. 